\title{
Maximum Density Space Packing with Parallel Strings of Spheres*
}

\author{
A. Bezdek ${ }^{1}$, W. Kuperberg ${ }^{1}$, and E. Makai ${ }^{2}$ \\ ${ }^{1}$ Division of Mathematics, F.A.T., Auburn University, \\ Auburn, AL 36849, USA \\ 2 Mathematical Research Institute of the Hungarian Academy of Sciences, \\ Realtanoda u 13 15, 1035 Budapest, Hungary
}

\begin{abstract}
A string of spheres is a sequence of nonoverlapping unit spheres in $\mathbf{R}^{3}$ whose centers are collinear and such that each sphere is tangent to exactly two other spheres. We prove that if a packing with spheres in $\mathbf{R}^{3}$ consists of parallel translates of a string of spheres, then the density of the packing is smaller than or equal to $\pi / \sqrt{18}$. This density is attained in the well-known densest lattice sphere packing. A longstanding conjecture is that this density is maximum among all sphere packings in space, to which our proof can be considered a partial result.
\end{abstract}

\section{Introduction}

Let $\mathscr{P}_{0}$ denote the lattice packing with unit spheres in $\mathbf{R}^{3}$ described as follows. Start with a pair of tangent unit spheres and a parallel translation of $\mathbf{R}^{3}$ which carries one of the spheres onto the other. The iterates of this translation and their inverses generate a sequence of unit spheres with disjoint interiors and collinear centers, and such that each of the spheres is tangent to exactly two others spheres. A sequence of spheres of this type we call a string of spheres (see Fig. 1). The line containing the centers of all spheres in one string will be called the axis of the string. Two strings are parallel if their axes are parallel. Next, consider a pair of nonoverlapping parallel strings such that the distance between their axes is $\sqrt{3}$, i.e., the minimum, and a parallel translation of $\mathbf{R}^{3}$ which carries one of the strings onto the other. The iterates of this translation and their inverses generate a sequence of strings, forming a collection of nonoverlapping unit spheres with coplanar centers, which we call a layer. Finally, consider a pair of parallel nonoverlapping layers as close to each

\footnotetext{
* The work of A. Bezdek and E. Makai was partially supported by the Hungarian National Foundation for Scientific Research under Grant Number 1238.
} 


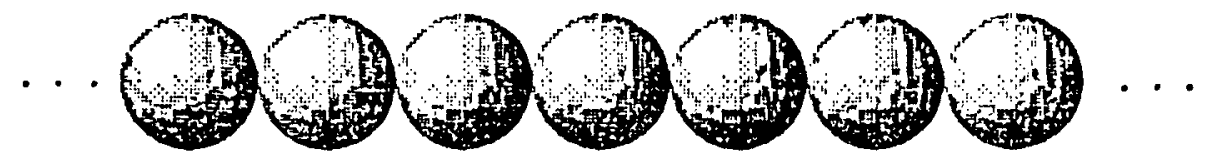

Fig. 1.

other as possible, and a parallel translation of $\mathbf{R}^{3}$ which carries one of the layers onto the other. The iterates of this translation and their inverses generate a sequence of layers. The resulting collection of unit spheres is the lattice packing $\mathscr{P}_{0}$. The density of this packing is $\pi / \sqrt{18}$, known to be the maximum among all lattice packings with spheres in $\mathbf{R}^{3}$ (see [6], [8], [4], and [5]). A long-standing conjecture states that this density cannot be exceeded in nonlattice packings either. For a survey of recent results concerning this and other problems in the theory of packing and covering, see [4]. The fact that $\mathscr{P}_{0}$ consists of parallel strings of spheres leads naturally to the following conjecture, stated by Böröczky [1] and Woods [9] independently: If a packing of $\mathbf{R}^{3}$ with unit spheres consists of parallel strings of spheres, then its density does not exceed $\pi / \sqrt{18}$.

The aim of this paper is to prove this conjecture.

\section{Preliminaries and Notation}

For the basic concepts of packing and density, the reader is referred to [5]. A packing with congruent copies of a set is saturated if no new congruent copy can be added to it without overlapping with any of the existing ones. Obviously, every packing can be extended to a saturated one, and this extension does not decrease its density.

Throughout this paper $\mathscr{P}$ denotes a packing of $\mathbf{R}^{3}$ with translates of a string of spheres, $\mathscr{P}=\left\{s_{1}, s_{2}, \ldots\right\}$. The axis of the string $s_{i}$ will be denoted by $t_{i}$. Since the aim of this paper is to prove that the density of $\mathscr{P}$ is at most $\pi / \sqrt{18}$, we can assume that $\mathscr{P}$ is saturated. Let $H$ be a plane perpendicular to the axes $t_{i}$ and let $P_{i}$ be the intersection point between $H$ and $t_{i}$. Notice that:

The distance between any two points of $\left\{P_{i}\right\}$ is greater than or equal to $\sqrt{3}$,

and

Any circle of radius 2 in $H$ contains at least one point of $\left\{P_{i}\right\}$.

By associating with each point $P_{i}$ the set $D_{i}$ of all points of $H$ lying nearer to $P_{i}$ than to any other point, we obtain a partition of $H$ into convex cells. Each of the cells $D_{i}$ is called a Dirichlet cell associated with the set $\left\{P_{i}\right\}$. The closure of each of these cells is a convex polygon. The collection of these polygons is referred to as the 
$D$-tiling of $H$ (see [3], Dirichlet tiling). Each member of the D-tiling is called a $D$ tile. Every point $P_{i}$ is contained in exactly one D-tile.

Another tiling of $H$ is obtained by connecting the points $P_{i}$ and $P_{j}$ with a line segment whenever their corresponding D-tiles have a common edge. This tiling, dual to the D-tiling, is called the $V$-tiling of $H$ (see [2], Voronoi-Delaunay tiling). Each member of the $\mathrm{V}$-tiling is called a $V$-tile. Every $\mathrm{V}$-tile is a polygon whose vertices lie in the set $\left\{P_{i}\right\}$. It is known (see [7]) that every V-tile is inscribed in a circle which contains in its interior no point of the set $\left\{P_{i}\right\}$.

Let $T$ be a $V$-tile. If $n$ is the number of vertices of $T$, then the density of the packing $\mathscr{P}$ relative to the infinite right prism over $T$ is

$$
d_{T}=\frac{n-2}{2} \frac{\operatorname{Vol}(S)}{2 \operatorname{Area}(T)}
$$

where $S$ is the unit sphere.

Thus the density of $\mathscr{P}$ can be determined by the areas of the V-tiles and the numbers of their sides.

In the next section we state and prove some lemmas whose purpose is to determine a relation between the areas and the numbers of sides of the $\mathrm{V}$-tiles.

\section{Lower Bounds for Areas of V-tiles}

In addition to the notation introduced thus far, we assume the following: $n$ denotes an integer greater than or equal to $3, r$ denotes a positive number, and $C$ is a circle of radius $r$.

Lemma 3.1. Among all n-gons inscribed in $C$ and with sides of length greater than or equal to a given number $a \leq 2 r \sin (\pi / n)$, the one which has $\mathrm{n}-1$ sides of length $a$ is of minimum area.

Proof. Suppose that the $n$-gon $p$ inscribed in $C$ is of minimum area among all $n$-gons inscribed in $C$ and with sides of length greater than or equal to $a$. (The existence of a minimum-area polygon among them is obvious.) Denote by $a_{0}$ a side of $p$ of maximum length, and let $a_{1}$ be the side of $p$ of maximum length among the remaining sides of $p$. Since the area of a polygon inscribed in a circle depends only on the lengths of its sides and not on their order, we may assume that the sides $a_{0}$ and $a_{1}$ are adjacent. Now, if $a_{1}$ is of length greater than $a$, then, by moving the vertex $a_{0} \cap a_{1}$ along $C$ so as to shorten the size of $a_{1}$ down to $a$, we obtain an $n$-gon $p^{\prime}$ of area smaller than that of $p$, while all sides of $p^{\prime}$ are still of length greater than or equal to $a$. Since this is impossible, for $p$ is of minimum area, the length of $a_{1}$ must be equal to $a$. Hence each side of $p$, with the possible exception of $a_{0}$, is of length $a$.

Lemma 3.2. Given an $n$-gon $P$ with sides $a_{1}, a_{2}, \ldots, a_{n}$, inscribed in a circle and containing the center of the circle, then, among all $n$-gons $Q$ whose sides $x_{1}, x_{2}, \ldots, x_{n}$ 
satisfy the inequalities $a_{i} \leq a_{i}(i=1,2, \ldots, n)$, the area of $P$ is maximum. Moreover, if $\operatorname{Area}(Q)=\operatorname{Area}(P)$, then $x_{i}=a_{i}$ for all $i$.

Proof. Among all $n$-gons $Q$ with sides $x_{i} \leq a_{i}$ there exists one of maximum area, denote it by $Q_{0}$ and denote its sides by $y_{i}(i=1,2, \ldots, n)$. By a well-known theorem [10], $Q_{0}$ is inscribed in a circle. Thus, the proof of the lemma is reduced to showing that $y_{i}=a_{i}$ for all $i$. Assume to the contrary that, say, $y_{1}<a_{1}$. Obviously, $Q_{0}$ is convex. Observe that if $v$ is a vertex of $Q_{0}$ which does not lie on $y_{1}$, then the angle at which $y_{1}$ is seen from $v$ must be right. Otherwise the area of $Q_{0}$ could be increased by changing that angle slightly to make it closer to $90^{\circ}$ while keeping $y_{1}$ shorter than $a_{1}$ and leaving all the other sides unchanged. This implies that all vertices of $Q_{0}$ lie on a circle whose diameter is $y_{1}$. Therefore $y_{i}=a_{i}$ for all $i>1$, because only one side of $Q_{0}$ can be the diameter of the circumscribed circle. Now, let $d$ denote the diameter of the circle in which $P$ is inscribed. Since $a_{1}>y_{1}$ and $d \geq a_{1}$, the circumcircle of $P$ is larger than that of $Q_{0}$, But since one side of $Q_{0}$ is the diameter of its circumcircle, and all the other sides of $Q_{0}$ are of the same length as the corresponding sides of $P$, this implies that $P$ does not contain the center of its circumcircle, contrary to the assumption.

Lemma 3.3. If $T$ is an $n$-sided $V$-tile $(n \geq 4)$, then $\operatorname{Area}(T)>(n-2) \sqrt{2}$.

Proof. Let $C$ be the circle with center $M$ and radius $r$, circumscribed about $T$. By (2.2), $r \leq 2$. By Lemma 3.1, Area $(T) \geq \operatorname{Area}\left(T_{n}(r)\right.$ ), where $T_{n}(r)$ denotes the $n$-gon inscribed in $C$ whose $n-1$ sides are of length $\sqrt{3}$ each and the remaining side is at least that long. Notice that if $n>4$, then $M$ lies in the interior of $T_{n}(r)$.

Case I. $M$ lies in the interior of $T_{n}(r)$. By Lemma 3.2, the area of $T_{n}(r)$ is greater than or equal to the area of a regular $n$-gon of side $\sqrt{3}$, which is $\frac{3}{4} n \cot (\pi / n)$. Thus, in this case, the proof is reduced to the inequality $\frac{3}{4} n \cot (\pi / n) \geq(n-2) \sqrt{2}$, which is equivalent to $n /(n-2) \cot (\pi / n) \geq 4 \sqrt{2 / 3}$. Since this last inequality holds for $n=4$ and its left-hand side increases with $n$, it is true for all $n \geq 4$.

Case 1I. $T_{n}(r)$ does not contain $M$. Then $n=4$ and $\operatorname{Area}\left(T_{4}(r)\right) \geq$ $\operatorname{Area}\left(T_{4}(2)\right)=\frac{39}{64} \sqrt{39}>2 \sqrt{2}$, and the proof is complete.

Lemma 3.4. If a $V$-tile $T$ is an acute triangle, then $\operatorname{Area}(T) \geq \sqrt{2}$.

Proof. Let $\Delta$ denote the (acute) triangle in $H$ with sides $\sqrt{3}, \sqrt{3}$, and 2 . Notice that $\operatorname{Area}(\Delta)=\sqrt{2}$ and notice that $\Delta$ can be obtained by an arrangement of three nonoverlapping strings perpendicular to $H$. Obviously, there exists an arrangement of three nonoverlapping strings perpendicular to $H$ that yields an acute triangle of minimum area (by acute traingle we mean here one whose every angle is of measure less than or equal to $90^{\circ}$ ). Denote such a minimum triangle by $T_{0}$. We shall show that $T_{0}$ is congruent to $\Delta$, which will prove this lemma. Let the vertices of $T_{0}$ be $p^{1}, p^{2}$, and $p^{3}$, defined by the strings $s^{1}, s^{2}$, and $s^{3}$, respectively. 
We prove first that the strings $s^{1}, s^{2}$, and $s^{3}$ touch each other. Obviously, two of the strings, say $s^{1}$ and $s^{2}$, must touch, otherwise the area of $T_{0}$ could be decreased by shortening all of its sides at the same ratio. Then, $s^{3}$ must touch either $s^{1}$ or $s^{2}$, otherwise the area of $T_{0}$ could be decreased by moving $s^{3}$ closer to the plane of the axes of $s^{1}$ and $s^{2}$. During this motion $T_{0}$ cannot become obtuse because the distance $p^{1} p^{2}$ is smaller than or equal to 2 , each of the distances $p^{1} p^{3}$ and $p^{2} p^{3}$ is always greater than or equal to $\sqrt{3}$, and $p^{3}$ moves perpendicularly toward the line segment $p^{1} p^{2}$. Let us say then that $s^{3}$ touches $s^{1}$. Now, if $s^{2}$ and $s^{3}$ did not touch, then again the area of $T_{0}$ could be decreased by reducing the angle between the sides $p^{1} p^{2}$ and $p^{1} p^{3}$. During this motion $T_{0}$ could not become obtuse because the sides $p^{1} p^{2}$ and $p^{1} p^{3}$ are of length of at least $\sqrt{3}$ and at most 2 , while $p^{2} p^{3}$ cannot become shorter than $\sqrt{3}$.

During the subsequent motions the three parallel strings will remain touching each other, therefore the resulting triangle will always remain acute. Indeed, in any obtuse triangle the ratio between its longest and its shortest side is greater than $\sqrt{2}$, but in every triangle obtained from three mutually touching strings that ratio is at most $2 / \sqrt{3}<\sqrt{2}$.

Now, since $T_{0}$ is of minimum area, each sphere from the string $s^{3}$ touches at least three spheres from the other two strings. Otherwise each sphere from $s^{3}$ would touch exactly two spheres from the other two strings, namely one from each, and that would make it possible to "slide" $s^{3}$ (keeping $s^{1}$ and $s^{2}$ fixed) closer to the plane of the axes of $s_{1}$ and $s_{2}$ thereby decreasing the area of $T_{0}$. Thus, a sphere from $s^{3}$ touches two spheres in one of the other strings, say $s^{1}$. This makes the distance $p^{1} p^{3}$ equal to $\sqrt{3}$. Now, for the same reason as above, a sphere from $s^{2}$ touches at least three spheres from the other two strings, say two of them are from $s^{1}$. This makes $p^{1} p^{2}=\sqrt{3}$ and, consequently, $p^{2} p^{3}=2$. In effect, $T_{0}$ and $\Delta$ are congruent, and the lemma is proved.

As we noted before, each V-tile is inscribed in a circle which contains no points from $\left\{P_{i}\right\}$ in its interior and therefore the circle is of radius smaller than or equal to 2. On the other hand, each edge of a V-tile is at least $\sqrt{3}$ long. This implies that the number of sides of each $\mathrm{V}$-tile is at most 7 . We are now concerned with those $\mathrm{V}$-tiles which are of area smaller than $\sqrt{2}$. For brevity, we call each such tile a singular tile. Observe that each singular tile is an obtuse triangle whose longest side is of length greater than $2 \sqrt{2}$, since the triangle with sides $\sqrt{3}, \sqrt{3}, 2 \sqrt{2}$ is of area $\sqrt{2}$. Also observe that the area of any singular tile is greater than or equal to $\sqrt{351} / 16=$ $1.170937 \ldots$, which is the area of a triangle with two sides of length $\sqrt{3}$ inscribed in a circle of radius 2. Denote this minimum area of a singular tile by $\delta$.

Lemma 3.5. If $T$ is an $n$-sided $V$-tile sharing the longest side of $k \geq 1$ singular tiles with them, then $\operatorname{Area}(T) \geq(n-2) \sqrt{2}+k(\sqrt{2}-\delta)$.

Proof. Since the square of side $2 \sqrt{2}$ has circumradius 2, no V-tile shares the longest side of more than three singular tiles with them. In other words, $k \leq 3$. Let $n$ 
denote the number of sides of $T, 3 \leq n \leq 7$. Let us consider the following cases:

(a) $n \geq 5$. By Lemma 3.2 the area of $T$ is greater than the area of a regular $n$-gon of side $\sqrt{3}$, i.e., $\operatorname{Area}(T)>\frac{3}{4} n \cot (\pi / n)>(n-2) \sqrt{2}+3(\sqrt{2}-\delta)$, which closes the case.

(b) $n=4$. Since the quadrilateral with consecutive sides of length $2 \sqrt{2}, \sqrt{3}$, and $\sqrt{3}$ and with circumradius 2 contains the center of its circumcircle, so does $T$. By Lemma 3.2, the area of $T$ is greater than that of the trapezoid with sides $\sqrt{3}, \sqrt{3}, \sqrt{3}$, and $2 \sqrt{2}$. The area of that trapezoid is equal to $\sqrt{4 \sqrt{6}+1}$ $(2 \sqrt{2}+\sqrt{3}) / 4=3.74646 \ldots$, which is greater than $2 \sqrt{2}+3(\sqrt{2}-\delta)=$ 3.55825 ...

(c) $n=3$. Let $T^{0}$ denote

(i) the equilateral triangle of side $2 \sqrt{2}$, if $k=3$;

(ii) the triangle of sides $\sqrt{3}, 2 \sqrt{2}$, and $2 \sqrt{2}$, if $k=2$;

(iii) the triangle with two sides $2 \sqrt{2}, \sqrt{3}$, and with circumradius 2 , if $k=1$ and $T$ is obtuse; or

(iv) the right triangle with sides $2 \sqrt{2}, \sqrt{3}$, and $\sqrt{5}$, if $k=1$ and $T$ is acute.

It is easy to see that $\operatorname{Area}(T)>\operatorname{Area}\left(T^{0}\right)$, and direct computations show that $\operatorname{Area}\left(T^{0}\right)>\sqrt{2}+k(\sqrt{2}-\delta)$.

This completes the proof of the lemma.

\section{Conclusion: The Density of the String Packing}

Theorem 4.1. The density of $\mathscr{P}$ is smaller than or equal to $\pi / \sqrt{18}$.

Proof. Let $T$ be a V-tile. The density $d_{T}$ of $\mathscr{P}$ relative to the infinite right prism over $T$ depends on the area and the number of sides of $T$, as stated in (2.3). Therefore, by Lemmas 3.3 and 3.4, if $T$ is nonsingular, then $d_{T} \leq \pi / \sqrt{18}$. If $T$ is singular, then, by Lemma 3.5 , across its longest side, $T$ has a nonsingular neighbour $T^{\prime}$ such that the density relative to the prism over $T^{\prime}$ is small enough to compensate for the "surplus" of that for the prism over $T$ and every other singular tile sharing its longest side with $T^{\prime}$. Thus we conclude that the density of $\mathscr{P}$ is smaller than or equal to $\pi / \sqrt{18}$.

\section{Related Problems and Remarks}

Remark. The result of this paper can be easily generalized to packings of parallel half-strings of spheres.

Problem 1. Generalize the result to packings of strings without the "parallel" assumption. 
Problem 2. Generalize the result to finite (of length $n$ ) strings of spheres, parallel or not necessarily parallel.

Problem 3. A cluster of spheres is a connected union of finitely many nonoverlapping unit spheres and such that the lattice packing $\mathscr{P}_{0}$ is the union of nonoverlapping congruent copies [translates] of it. Produce a small cluster of spheres whose packing density with its congruent copies [translates] can be proved to be equal to $\pi / \sqrt{18}$.

Remark on Problem 3. It is not difficult to construct a large cluster of spheres whose packing density with its translates is equal to $\pi / \sqrt{18}$. The meaning of Problem 3 is to construct a reasonably small cluster of spheres with this property. An example of such a cluster of fewer than 15 spheres would be of interest and a proof for a cluster of two spheres would be a very significant result.

\section{References}

1. Böröczky, K., Research Problem 12., Period. Math. Hungar, 6. (1) (1975), 109.

2. Delaunay, B., Sur la sphere vide, Bull. Acad. Sci. URSS, Cl. Sci. Mat. Natur., VII (1934), 793-800.

3. Dirichlet, G. L., Uber die Reduktion der positiven quadratische Formen mit drei unbestimmten ganzen Zahlen, J. Reine Angew. Math., 40 (1850), 209-227.

4. Fejes Tóth, G., New results in the theory of packing and covering, in Convexity and Its Applications, P. M. Gruber and J. M. Wills, ed., Birkhäuser, Basel, 1983, pp. 318-359.

5. Fejes Tóth, L., Lagerungen in der Ebene, auf der Kugel und in Raum, 2nd Auflage, Springer-Verlag, Berlin, 1972.

6. Gauss, C. F., Untersuchungen über die Eigenschaften der positiven ternaren quadratischen Formen von Ludwig August Seeber, Göttingische gelehrte Anzeigen, 1831 Juli $9=$ J. Reine Angew. Math. 20 (1840), 312-320 = Werke, Vol. 11, pp. 188-196.

7. Molnár, J., On the $\rho$-system of unit circles, Ann. Univ. Sci. Budapest. Sect. Math. 20 (1977), 195-203.

8. Rogers, C. A., Packing and Covering, Cambridge University Press, Cambridge, 1964, pp. 1-5.

9. Woods, A. C., Private communication.

10. Yaglom, I. M., Boltyanskiy, V. G., Convex Figures, Holt, Rinehart and Winston, New York, 1961.

Received February 22, 1989. 